\title{
Intermittent heart rate-dependent early repolarization pattern (J-point ST elevation) demonstrated on Holter recordings
}

\author{
Shlomo Stern
}

Emeritus Professor, The Hebrew University of Jerusalem, Jerusalem, Israel

\begin{abstract}
The J-point elevation of the ST segment, the early repolarization (ER) pattern is diagnosed usually on a resting 12-lead ECG. We investigated the ER pattern on 24-h Holter recordings under physiological conditions in 9,200 consecutive cases. The patients' age varied between 14 days to 95 years. In 410 individuals (4.9\% of the total) during at least one period of the 24 -h recording $J$-point ST elevation of $\geq 1 \mathrm{~mm}$ was found in $\geq 2$ of the 3 Holter leads, lasting for $\geq 3$ min. In 382 patients (93.1\% of the 410 with ER), the typical ER pattern was intermittent, i.e., seen only below heart rate (HR) of about 70/min, and as the HR climbed, beyond 65-75/min, the ER pattern disappeared and then reappeared when the HR slowed down again. This was observed during the $24 \mathrm{~h}$ period several times, usually 10-20 times during the 24- $h$ period. In only 28 examinees (6.9\% of the 410 cases with $E R, 0.33 \%$ of the total 8,300 examinees included in the study) this pattern prevailed at HR faster than 75/min. Thus, if an ER pattern is seen on a "spot"-ECG recorded at slow HR, this does not ensure that this pattern will not disappear when HR rises. On the other hand, if no ER pattern is seen in a 12-lead ECG recorded on a relatively higher $H R$, we demonstrated that this pattern may temporarily appear when HR slows down. (Cardiol J 2014; 21, 2: 198-201)
\end{abstract}

Key words: early repolarization, bradycardia — dependent, Holter monitoring

\section{Introduction}

A slow heart rate (HR) as an exaggerator of the J-point elevation of the ST segment seen on the 12-lead ECG, the early repolarization (ER) pattern, has been mentioned in previous investigations, explaining thus the enhancement of this pattern in athletes and the nocturnal occurrence of ER-associated sudden cardiac death. In their pioneering work Haissaguerre et al. [1] evaluated "baseline electrocardiograms for the presence of early repolarization" [2] and found it to be a bad prognostic sign. Rosso et al. [2] in the same years used also one "spot" ECG for the diagnosis of the presence or the absence of ER pattern, as did other investigators. All these groundbreaking studies
$[3,4]$ provide no information on the presence or absence of ER pattern on the "spot" ECG and no ECG tracing with HR different, faster or slower, than the HR on the "spot ECG". Conversely, we used continuous 24-h monitoring method to look for the prevalence of ER pattern at different heart rates under physiological condition.

\section{Methods}

In this prospectively designed study we investigated 9,200 consecutive Holter recordings for the presence or absence of ER pattern during 24-h period. ER pattern was diagnosed if an elevation of the QRS-ST junction (J point) was found in at least 2 leads. The amplitude of J-point elevation had to 


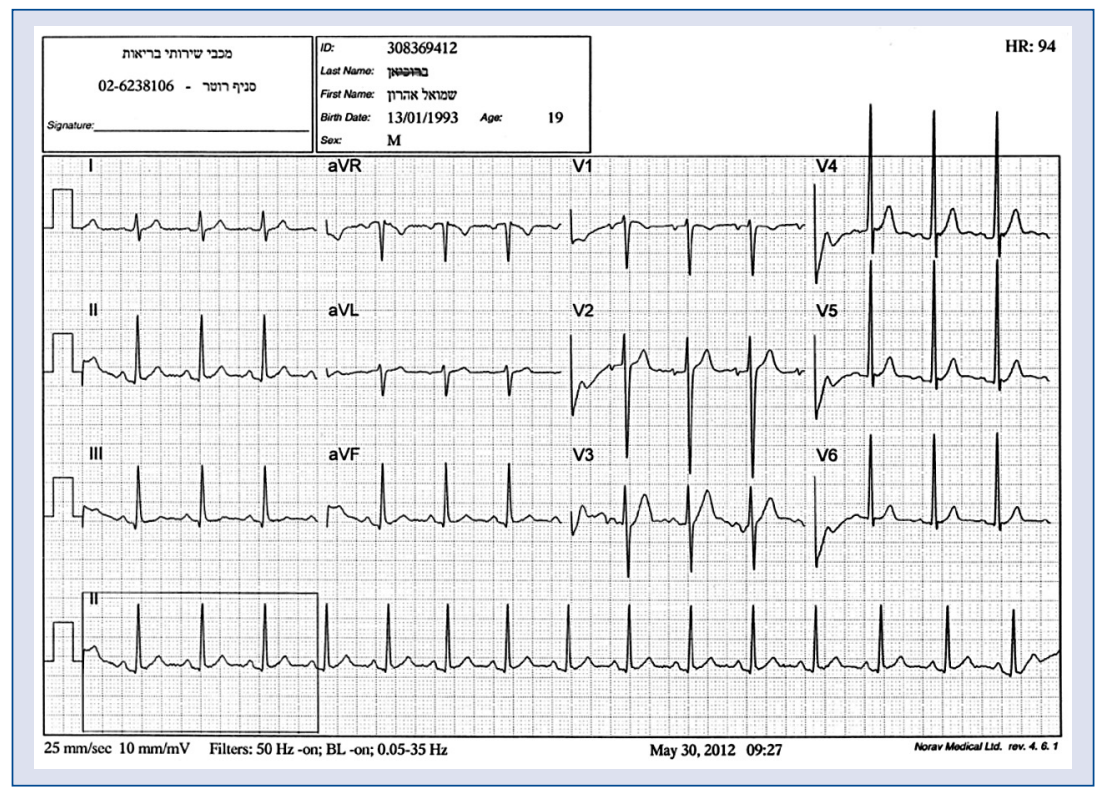

Figure 1. Resting 12-lead ECG of a 19-year-old male, complaining of palpitations. Early repolarization (ER) was 105/min at the time of the recording; note absence of the ER pattern.

be at least $1.0 \mathrm{~mm}, 1 \mathrm{mV}$ above the baseline level, either as QRS slurring (a smooth transition from the QRS segment to the ST segment) or notching (a positive $\mathrm{J}$ deflection inscribed on the $\mathrm{S}$ wave) in the inferior lead, lateral lead, or both, the PR interval we used as the isoelectric line, a concave ST elevation was $>0.1 \mathrm{mV}$ requested and the terminal part of the QRS complex might have been slurred.

The age of the examinees varied from 2 weeks to 95 years, all having been referred to the Assuta Telemedicine Center, Telaviv, Israel. The interpretation system was the Pathfinder produced by Spacelabs (formerly Reynolds DelMar). The pathfinder activates 3 -channelrecorder with 4 electrode patient cables. The data is then collected with software created by eWave (Telaviv, Israel) which subsequently enables fast transmission to the interpretation center and back to the referring center. The data were first screened and visually checked by trained technicians, and later checked by an experienced physician.

The cause of referral was in $58 \%$ subjectively felt "palpitations" without previous documentation of an arrhythmia, in 11\% a follow-up for previously diagnosed atrial fibrillation or other supraventricular tachycardia, in $5 \%$ there were previously documented ventricular arrhythmias, mainly ventricular premature beats, $10 \%$ were referred for ischemia evaluation and $5 \%$ for dizziness or other unspecified reasons. All recordings suspected for J-point ST elevation were scrutinized again, printed out and the diagnosis of ER was confirmed on the printed version.

\section{Results}

After having excluded records with significant supraventricular and ventricular arrhythmias, conduction disturbances or ischemic changes, a total of 8,300 normal sinus rhythm recordings were analyzed on-line at least $3 \mathrm{~min}$. In $382 \mathrm{pa}-$ tients (93.1\% of the 410 with ER), the typical ER pattern was seen only below HR of $70 / \mathrm{min}$, while as the HR spontaneously climbed beyond 65-75/ $/ \mathrm{min}$, the ST-segment became isoelectric and the ER pattern disappeared. In these 382 patients the presence of ER at a slow HR, its disappearance at a faster HR and reappearance whenever the HR dropped again below $60-70 / \mathrm{min}$, were observed during the 24-h period on an average of 11 times, ranging between 10-20 times (Fig. 1, 2). In only 28 examinees ( $6.9 \%$ of the 410 with ER, $0.33 \%$ of the total of 8,300 examinees) this pattern prevailed with HR faster than $75 / \min$ (Table 1).

\section{Discussion}

In most previous investigations the diagnosis of ER pattern was based on a "spot" resting 12-lead ECG, and therefore the dependence of this pattern in an overwhelming majority of the cases on the HR, was not described. Evaluating nearly 


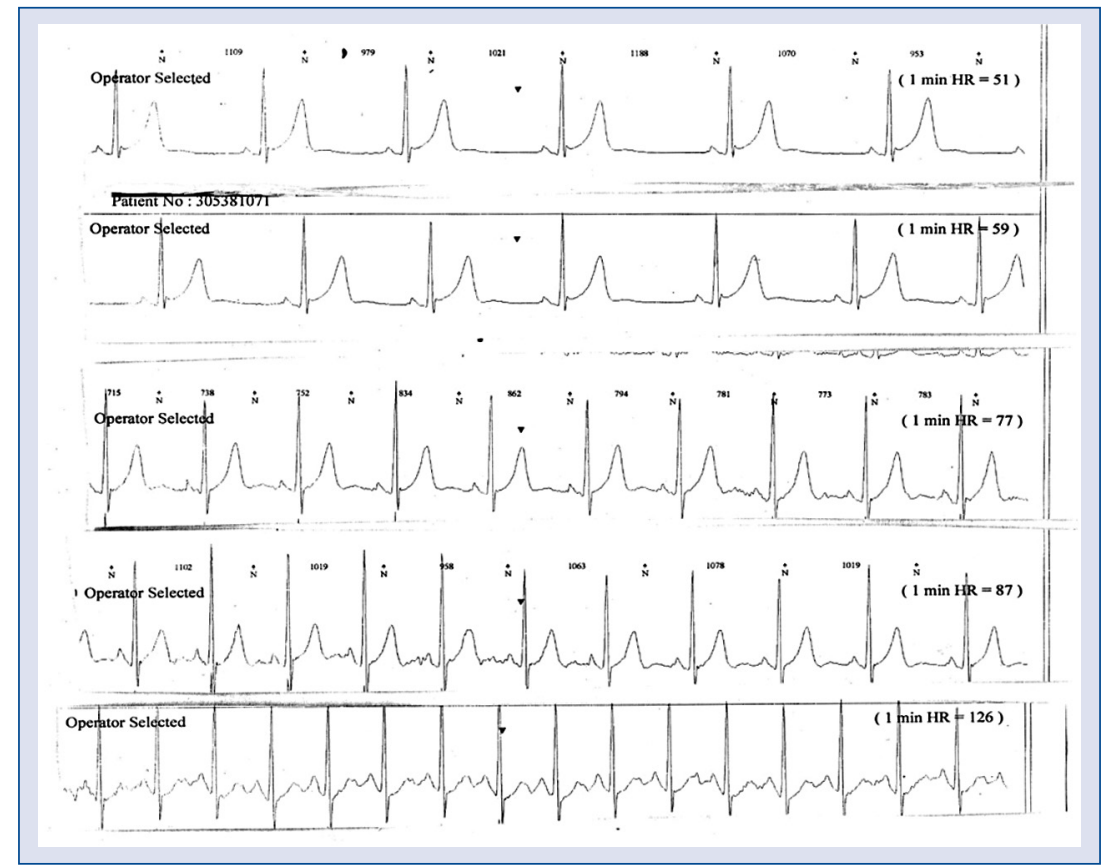

Figure 2. The same patient's 24-h Holter recording, started the same day as the ECG in Figure 1 was performed; note early repolarization pattern at the heart rate (HR) of $51 / \mathrm{min}$ and $59 / \mathrm{min}$ and its absence at $\mathrm{HR}$ at $77 / \mathrm{min}$ or faster.

Table 1. Early repolarization according with the heart rate.

\begin{tabular}{|c|c|c|c|c|}
\hline & Numbers & Percent & $\begin{array}{l}\text { In the group of } \\
410 \text { with ER }\end{array}$ & $\begin{array}{c}\text { In total } 8,300 \\
\text { examinees }\end{array}$ \\
\hline Total number of examinees & 9,200 & & & \\
\hline After exclusions (see text) & 8,300 & $90.21 \%$ & & \\
\hline ER pattern (see text) & 410 & $4.9 \%$ & & \\
\hline ER pattern seen below HR of $70 / \mathrm{min}$ & 410 & $4.9 \%$ & & \\
\hline ER disappearing at HR beyond $65-75 / \mathrm{min}$ & 382 & & $93.1 \%$ & $4.15 \%$ \\
\hline ER persistent at faster HR as well & 28 & & $6.9 \%$ & $0.33 \%$ \\
\hline
\end{tabular}

$\mathrm{ER}$ - early repolarization; $\mathrm{HR}$ - heart rate

30,000 Holter recordings, Uberoi et al. [5] found in ambulatory clinical population the ER pattern in the lateral, inferior or both leads at an HR of between $63-66 / \mathrm{min}$, as compared to the average HR of 70/min of all the subjects. In athletes Noseworthy et al. [6] diagnosed ER pattern at an average HR of $57.5 \pm 8.7$, while HR was $61.2 \pm 9.9$ in those without the ER pattern $(\mathrm{p}<0.001)$. The same authors found in a general population a non-significantly slower HR in those with ER patterns, in both genders [7]. In the investigation of Cappato et al. [8] athletes with J wave/QRS slurring had a HR of $57 \pm 5$, as compared to $61 \pm 7$ in those without. In the Pilot Finnish Athlete Population study HR was lower in subjects with ER
$(68 \pm 1)$ than in those without $(71 \pm 1, \mathrm{p}=0.02)$. In athletes in the Miami Validation Population: in the general population HR was $70 \pm 1$ in subjects with and $76 \pm 1$ in those without an ER pattern, a difference statistically significant only in patients with an ascending/upsloping ST-segment.

\section{Limitations of the study}

This study, using the generally accepted 3 -channel Holter recording, is not a source for characterization of the ER pattern, even though it uses the commonly accepted criteria for the diagnosis of this pattern. Furthermore, as no follow-up date is available in our study, no conclusion can be drawn as to whether this intermittent HR dependent type 
of ER has a different prognostic significance than an ER pattern which prevails also at higher HR. Only a long term prospective study using repeat or continuous ECG recordings could shed light on this matter.

\section{Conclusions}

We prove in this study that the appearance of ER pattern in the vast majority has a clear association with the HR during physiological day/night activities. Thus, the presence or absence of ER pattern on a "spot-ECG" recording may not always be enough to confirm or dismiss this diagnosis. This pattern most frequently prevails at a slow $\mathrm{HR}$ and may disappear even several minutes later, when the HR rises. This type of ER should be called an "intermittent ER pattern", and obviously can be observed when not only a single "spot" but a continuous ECG Holter recording is being scrutinized for diagnosis. This is in line with the suggestion of Prior et al. [9] that "there are no validated techniques to provoke the ER pattern, although 12-lead Holter monitoring to detect evidence of the ER pattern during bradycardia is warranted".

\section{Acknowledgements}

The excellent secretarial help of Ms. Estelle Rachamim-Rayman is greatly acknowledged.

Conflict of interest: none declared

\section{References}

1. Haissaguerre M, Derval N, Sacher F et al. Sudden cardiac arrest Associated with early repolarization. New Engl J Med, 2008; 358 : 2016-2013.

2. Rosso R, Kogan E, Belhassen B et al. J-point elevation in survivors of primary ventricular fibrillation and matched control subjects: Incidence and clinical significance. J Am Coll Cardiol, 2008; 52: 1231-1238.

3. Tikkanen JT, Juntitila J, Anttonen O et al. Early repolarization electrocardiographic phenotypes associated with favorable long-term outcome. Circulation, 2011; 123: 2666-2673.

4. Lanza GA, Mollo R, Cosenza A et al. Prevalence and clinical correlates of early repolarization and J wave in a large cohort of subjects without overt heart disease. J Electrocardiol, 2012; 45: 404-410.

5. Uberoi A, Jain NA, Perez M. Early repolarization in an ambulatory clinical population. Circulation, 2011; 124: 2208-2214.

6. Noseworthy PA, Weiner R, Kim J et al. Early repolarization pattern in competitive athletes: Clinical correlates and the effects of exercise training. Circ Arrhythm Electrophysiol, 2011; 4: 432-440.

7. Noseworthy PA, Tikkanen JT, Portham K et al. The early repolarization pattern in the general population: clinical correlates and heritability. J Am Coll Cardiol, 2011; 57: 2284-2289.

8. Cappato R, Furlanello F, Giovinazzo V et al. J wave, QRS slurring, and ST elevation in athletes with cardiac arrest in the absence of heart disease: Marker of risk or innocent bystander? Circ Arrhythm Electrophysiol, 2011; 3: 305-311.

9. Prior SG, Wilde AA, Horie M, Cho Y. HRS/EHRA/APHRS expert consensus statement on the diagnosis and management of patients with inherited primary arrhythmia syndromes. This document was embargoed until May 10, 2013 at 9.30 am MSR. Document will be published in Heart Rhythm, EP Europace, and Journal of Arrhythmias in the fall of 2013. 